\title{
LA INFLUENCIA DE UN PROGRAMA DE ACTIVIDAD FÍSICA SOBRE PARÁMETROS CORPORALES Y CARDIOVASCULARES EN MUJERES PREMENOPÁUSICAS
}

\author{
Almudena Ramírez Balas, Diego Muñoz Marín, Rafael Timón \\ Andrada y Guillermo J. Olcina Camacho ${ }^{1}, \quad M^{a}$ Concepción \\ Robles Gil $^{2}$ y Marcos Maynar Mariño ${ }^{3}$ \\ ${ }^{1}$ Facultad de Ciencias del Deporte. Universidad de \\ Extremadura, España \\ ${ }^{2}$ Departamento de Didáctica de la Expresión Musical, Plástica y \\ Corporal. Facultad de Ciencias del Deporte de Cáceres. \\ Universidad de Extremadura \\ ${ }^{3}$ Departamento de Fisiología. Facultad de Ciencias del Deporte \\ de Cáceres. Universidad de Extremadura \\ alraba015@hotmail.com
}

RESUMEN

\begin{abstract}
Durante el ciclo de la vida, la figura corporal de la mujer se modifica paulatinamente, pero al llegar la edad mediana, a consecuencia de la pérdida folicular de la función ovárica entre otros factores, se acentúan los cambios fisiológicos que pueden aumentar el riesgo para la salud y el bienestar. La androgenia, en asociación con la obesidad, aumenta el riesgo de padecer diabetes, hipertensión, dislipidemia, arterosclerosis, enfermedades cardiovasculares, colelitiasis, artrosis, artritis, carcinomas femeninos y otras patologías de la edad mediana. El ejercicio físico ha adquirido cada vez mayor importancia dentro de la terapéutica en el climaterio. Se han demostrado los efectos positivos del ejercicio sobre las enfermedades cardiovasculares y el mantenimiento del peso. Por todo ello, el estudio pretende conocer la influencia de un programa de actividad física de cinco meses de duración sobre factores de riesgo cardiovasculares como la hipertensión arterial y la composición corporal en mujeres premenopáusicas. Participaron 20 mujeres premenopáusicas, separadas en dos grupos de mujeres: menores de 35 años $(n=10)$, y mayores de 35 años $(n=10)$. Las valoraciones previas y al finalizar el programa fueron: valoración de la tensión arterial y de la composición corporal. Los resultados mostraron una reducción significativa en mayor medida en la tensión arterial diastólica y en la masa grasa en el grupo de mujeres mayores de 35 años premenopáusicas. Por ello, practicar un programa de ejercicio físico basado en el aeróbic, clases de step y tonificación con mancuernas, mejora factores de riesgo cardiovasculares como la hipertensión arterial y la composición corporal en mujeres adultas mayores de 35 años premenopáusicas.
\end{abstract}

PALABRAS CLAVES: masa grasa, tensión arterial, aeróbic, índice de masa corporal, peso

\begin{abstract}
During the life cycle of a woman's body figure is changed gradually, but on reaching middle age, as a result of follicular loss of ovarian function among others, are emphasized the physiological changes and increase health risk and welfare. Androgyny in association with obesity increases the risk for diabetes, hypertension, dyslipidemia, atherosclerosis, cardiovascular disease, gallstones, osteoarthritis, arthritis, cancers and other disorders of female middle age. Physical activity has gained importance increasing in therapy in the climacteric. It has demonstrated the positive effects of exercise on cardiovascular disease and weight maintenance. This study investigates the influence of a physical activity program for five months on cardiovascular risk factors like high blood pressure and body composition in premenopausal women. The study included 20 premenopausal women, separated into two groups: younger than 35 years $(\mathrm{n}=10)$, and over 35 years $(\mathrm{n}=10)$. The experimental subjects underwent an assessment of blood pressure and body composition before and after an aerobic training. Results indicate greater extent in reducing diastolic blood pressure and body fat in over 35 years premenopausal women. Therefore, performing aerobics, step and toning improves factors cardiovascular risk as high blood pressure and body composition in over 35 premenopausal women.
\end{abstract}

KEY WORDS: fat mass, blood pressure, aerobics, body mass index, weight 


\section{INTRODUCCIÓN:}

En relación con el biotipo y climaterio femenino, según Díaz Sánchez (1999), durante la edad adulta de la mujer se incrementa el exceso de grasa central que usualmente coexiste con un aumento de su condición mórbida. La función ovárica normal tiende a acumular más grasa alrededor de las caderas y los glúteos que en la región abdominal, aunque algunas exhiben un patrón superior ya desde la premenopausia. Con el advenimiento del climaterio femenino este morfotipo es más evidente, y se asocia a la declinación de la producción de estrógenos. El déficit de la producción estrogénica asociada a la disminución de la función ovárica, produce alteraciones a nivel fisiológico y morfológico. Con la edad la mujer cambia su composición corporal: pérdida de masa ósea, fracciones minerales en el hueso e incremento de la grasa total, visceral y fluido extracelular. Todas estas manifestaciones se incrementan con la menopausia.

Por lo tanto, durante el ciclo de la vida la figura corporal de la mujer se modifica paulatinamente, pero al llegar la edad mediana, a consecuencia de la pérdida folicular de la función ovárica entre otros factores, se acentúan los cambios fisiológicos que pueden aumentar el riego para la salud y el bienestar. La androgenia en asociación con la obesidad, aumenta el riesgo de padecer diabetes, hipertensión, dislipidemias, arterosclerosis, enfermedades cardiovasculares, colelitiasis, artrosis, artritis, carcinomas femeninos y otras patologías de la edad mediana (Hauner, 1995).

Actualmente se ha demostrado que la obesidad y la falta de ejercicio físico en mujeres contribuyen de manera independiente al desarrollo de diabetes tipo 2 (Rana, Li, Manson, y Hu, 2007), a un mayor riesgo de enfermedad coronaria (Arsenault y col., 2009), una mayor prevalencia de factores de riesgo cardiovasculares (Henry-Okafor y cols., 2011), así como el aumento de sufrir la enfermedad divercular (Hjern, Wolk, y Hakansson, 2011).

Además, los factores del estilo de vida que intervienen directamente en el entorno de la mujer guardan relación con sus características físicas. La distribución de la adiposidad se asocia a un bajo nivel de actividad física y educacional, consumo de alcohol, hábito de fumar e historia de infertilidad, entre otras características (Kaye, Folsom, Prineas, Potter, y Gapstur, 1990).

Los estudios que han investigado sobre la adiposidad en mujeres, indican que una alta adiposidad abdominal (índice cintura/cadera) se asocia con un riesgo de sufrir enfermedades cardiovasculares en mujeres mayores obesas y no obesas (Montalcini, Gorgone, Garzaniti, Gazzaruso, y Pujia, 2010); y con el accidente cerebro vascular isquémico en mujeres adultas (Kizer y cols., 2010).

Un estudio demuestra que los hábitos perjudiciales para la salud, entre ellos, el sedentarismo, se están convirtiendo en hechos cada vez más precoces en la vida de las personas. Además, este comportamiento negativo se intensifica más en los adultos jóvenes (Leyk y col., 2008). Por esto, el ejercicio físico ha adquirido cada vez mayor importancia dentro de la terapéutica en el climaterio, dado que se ha demostrado que por sí solo puede afectar a los factores de riesgo de las enfermedades cardiovasculares. Se han demostrado los efectos positivos del ejercicio sobre las enfermedades cardiovasculares (Cowley y col., 1992) y el mantenimiento de peso (Owens, Matthews, Wing, y Kuller, 1990). 
En estudios recientes se demuestran los beneficios de un estilo de vida activo, como realizar ejercicio regular mantiene o mejorar la salud funcional y mental en adultos mayores (Wang y cols., 2010); así como llevar a cabo un programa de ejercicio estructurado centrado en la resistencia, fuerza, flexibilidad y ejercicio de equilibrio postural mejora la capacidad cardiorrespiratoria y muscular en adultos (Van Roie y cols., 2010). Por todo ello, llevar un estilo de vida no saludable, como consumir tabaco, tener una dieta deficiente y no realizar ejercicio físico, incrementan el riesgo de sufrir enfermedades crónicas cardiovasculares, ciertos tipos de cáncer, diabetes y enfermedades pulmonares crónicas (Anthony y cols., 2012).

La actividad física regular puede reducir el riesgo de la muerte prematura por enfermedades coronarias, hipertensión, diabetes tipo II, cáncer de colon y mama y depresión (Godfrey y Nelson, 2009).

En la tabla 1 que se muestra a continuación, se pueden ver los efectos de distintos programas de actividad física:

Tabla 1.

Estudios de diferentes programas de actividad física en mujeres premenopáusicas

\begin{tabular}{|c|c|c|c|}
\hline Referencia & $\begin{array}{c}\text { Muestra } \\
\text { Experimental }\end{array}$ & Diseño del Programa & Resultados \\
\hline Mosher, Ferguson, y & 53 mujeres & 2 entrenamientos step: & En ambos grupos: \\
\hline Arnold (2005) & 18-23 años. & $\begin{array}{l}\text { Interválico/Continuo; } \\
12 \text { semanas, } \\
3 \text { días/semana, } \\
50 \text { min. duración. } \\
70 \text { - } 85 \text { \% FC Máxima. }\end{array}$ & $\begin{array}{l}\downarrow \% \mathrm{MG}^{*} ; \\
\uparrow \text { Condición } \\
\text { cardiovascular* }\end{array}$ \\
\hline Kin Isler, Kosar, y & 45 mujeres sedentarias & 2 programas aeróbic: & El aeróbic con step: \\
\hline Korkusuz (2001) & $\begin{array}{l}\text { sanas. } \\
\text { Estudiantes, jóvenes. }\end{array}$ & $\begin{array}{l}\text { Baile/aeróbic Step. } \\
8 \text { semanas, } \\
3 \text { días/semana, } \\
45 \text { min. duración } \\
60 \text {-70 \% de FC Máxima. }\end{array}$ & $\downarrow$ perfiles lipídicos* \\
\hline Isler (2006) & $\begin{array}{l}\text { Mujeres } \\
\text { premenopaúsicas. }\end{array}$ & $\begin{array}{l}2 \text { programas diferentes: } \\
\text { Aeróbic step } \\
\text { Interválico/Continuo } \\
12 \text { semanas }\end{array}$ & $\begin{array}{l}\text { En ambos grupos: } \\
\uparrow \mathrm{CCR} \text { y mejora la CC* }\end{array}$ \\
\hline Kang, Matsuo, y & 11 mujeres. & Ejercicio de FR & $\downarrow$ Peso corporal* \\
\hline Suzuki (2004) & 38 - 59 años. & $\begin{array}{l}\text { Mancuernas }(0,5 \text { - } 1 \text { kg.) } \\
\text { y bandas elásticas } \\
12 \text { semanas, } \\
3 \text { días/semana, } \\
40 \text { min. duración. }\end{array}$ & $\downarrow \% \mathrm{MG}^{*}$ \\
\hline $\begin{array}{l}\text { Kalman, Colker, } \\
\text { Wilets, Roufs, y } \\
\text { Antonio (1999) }\end{array}$ & $\begin{array}{l}\text { Personas con sobrepeso. } \\
2 \text { grupo: } \\
\text { - Grupo que tomaron } \\
\text { piruvato (6g/día). } \\
\text { - Grupo control con } \\
\text { placebo. }\end{array}$ & $\begin{array}{l}\text { Programa ejercicio físico } \\
\text { Aeróbico/Anaeróbico } \\
6 \text { meses duración } \\
3 \text { días/semana, } \\
\text { 45-60 min. duración }\end{array}$ & $\begin{array}{l}\text { Grupo piruvato: } \\
\downarrow \text { peso corporal* } \\
\downarrow \mathrm{MG}^{*} \\
\downarrow \% \mathrm{MG}^{*} \\
\text { Grupo con placebo: Ns en } \\
\text { la CC }\end{array}$ \\
\hline
\end{tabular}

CC: Composición Corporal; CCR: Condición Cardiorrespiratoria; FR: Fuerza Resistencia; MG: Masa Grasa; MLG: Masa Libre de Grasa. Ns: No significativo.

* Las flechas muestras cambios significativos

El objetivo del presente estudio es conocer la influencia de un programa de actividad física de cinco meses de duración, sobre factores de riesgo cardiovasculares como la hipertensión arterial y la composición corporal en mujeres premenopáusicas. 


\section{METODOLOGIA:}

\section{Muestra experimental:}

En el estudio participaron mujeres premenopáusicas $(n=20)$, separadas en dos grupos de diez mujeres: menores de 35 años $(n=10)$ y mayores de 35 años $(n=10)$.

Las características de la muestra se recogen en la tabla 2:

Tabla 2

Características iniciales de la muestra experimental

\begin{tabular}{lccc}
\hline Mujeres Premenopáusicas & $\begin{array}{c}\text { Menores de 35 años } \\
(\mathbf{n = 1 0 )}\end{array}$ & $\begin{array}{c}\text { Mayores de 35 años } \\
(\mathbf{n = 1 0})\end{array}$ & Total (n=20) \\
\hline Edad (años) & $24,89 \pm 4,88$ & $43,75 \pm 7,27$ & $30,69 \pm 10,54$ \\
Talla (cm) & $161,56 \pm 0,08$ & $159,50 \pm 0.05$ & $160,92 \pm 0,07$ \\
Peso (kg) & $60,27 \pm 10,71$ & $62,95 \pm 3,83$ & $61,10 \pm 9,04$ \\
\% Grasa & $23,55 \pm 8,01$ & $31,70 \pm 3,83$ & $26,06 \pm 7,86$ \\
\% Masa Libre Grasa & $76,44 \pm 8,01$ & $68,30 \pm 3,83$ & $73,93 \pm 7,86$ \\
\hline
\end{tabular}

Cada una de las mujeres firmó un consentimiento informado, cumpliendo los principios de la declaración de Helsinki y sus revisiones posteriores para estudios en humanos. De esta forma se garantiza la confidencialidad de los datos, entre otros. Las muestras serán anonimizadas, asegurando la imposibilidad de inferir su identidad, para su estudio y potencial análisis ulterior.

Como criterios de inclusión en el estudio hay que señalar: no tener historia de cáncer, no padecer diabetes mellitus, no tener desórdenes endocrinos y no tomar medicación que pudiera afectar a los parámetros del estudio.

\section{Diseño experimental:}

Para el estudio, se estableció un programa de intervención de aeróbic, clases de step y tonificación de 5 meses de duración, con una frecuencia semanal de 3 días alternos y con una duración de 70 minutos cada sesión a una intensidad entre el $60-70 \%$ de la frecuencia cardíaca máxima de reserva. Cada sesión estaba estructurada de la siguiente forma:

Un calentamiento, en el que se realizan estiramientos de los principales grupos musculares implicados, así como ejercicios de movilidad articular. Esta parte de la sesión tiene una duración entre 5-7 minutos.

Un período de actividad física moderada entre 40-45 minutos. La actividad física desarrollada era diferente dependiendo del día: los lunes se realizaban coreografías de aeróbic; los miércoles, una coreografía con step y los viernes se ejecutaban coreografías de aeróbic con la intensificación del movimiento de brazos con dos mancuernas de $1 / 2 \mathrm{~kg}$ o $1 \mathrm{~kg}$. Las coreografías eran más cortas y se introducían ejercicios de glúteos, piernas, pecho y brazos.

Una penúltima parte de tonificación de abdominales y lumbares, con una duración de 10 minutos.

Una última parte de vuelta a la calma, en la cual se realizaban ejercicios de relajación y estiramientos. Esta fase tenía una duración entre los 5 -7 minutos.

Los sujetos experimentales fueron sometidos a unas valoraciones tanto previas a la realización del programa, como al finalizar el mismo: historia clínica y deportiva, valoración de la tensión arterial y valoración de la composición corporal. 
Para evaluar la tensión arterial fue utilizado un tensiómetro Omron 7051T (HEM-759-E), Intelli sense. Los resultados que se obtienen son: tensión arterial sistólica en $\mathrm{mmHg}$ en posición sentada; tensión arterial diastólica en mmHg en posición sentada; y frecuencia cardiaca (en reposo) en posición sentada expresada en pulsaciones por minuto (ppm).

El protocolo empleado fue indicar a los sujetos que permanecieran sentados unos minutos previos a la medición. No se debía haber consumido bebidas alcohólicas ni productos a base de tabaco ni cafeína durante los 30 minutos previos a la medición. El brazo derecho se colocaba sobre una mesa al nivel del corazón, ligeramente flexionado, con la palma de la mano hacia arriba. El investigador colocaba el borde inferior del manguito a 2,5 cm por encima de la articulación del codo. Una vez realiza la medición, se descansaba un minuto y se procedía a realizar la medición dos veces más. Se anotaba el valor medio de las últimas dos mediciones.

El protocolo seguido consistió en decir al ejecutante que se desprendiera del calzado. Se le colocó de espaldas a la regla y mirando horizontalmente hacia delante. La espalda debía estar en contacto con la regla del tallímetro que tenía detrás. El que registró los datos debió comprobar que el cursor estaba totalmente horizontal con respecto al suelo para así registrar la altura en centímetros y milímetros.

El peso corporal se obtuvo a través de una báscula de precisión de 50 gr. marca Seca. Se calibró la báscula antes de comenzar a registrar los pesos, para ello la báscula fue colocada sobre un plano horizontal. La valoración del peso se registró en kilogramos $(\mathrm{kg})$. Para su valoración el sujeto se sube a la báscula descalzo, con la mínima ropa posible. Debido al lugar donde se realizó el registro de datos, se recomendó que la medición se realizase en pantalón corto y con una camiseta ligera. La persona se mantuvo inmóvil durante unos segundos hasta que la báscula fijara el peso, y así se registró el peso del sujeto en kg y gr.

Para evaluar la composición corporal se empleó la técnica de bioimpedancia. El material utilizado fue: Tanita Body Composition Analyzer BF-350. Max=200kg/440 Ib, y el programa informático: Suite Biológica 7 (Versión 7.0.1.201) - Suite Biologica Lite. Los datos obtenidos de la bioimpedancia fueron: peso (kg); masa libre de grasa (kg y \%); masa grasa (kg y \%); agua corporal (kg y \%); e índice de masa corporal $\left(\mathrm{kg} / \mathrm{m}^{2}\right)$.

El protocolo de medida seguido para la bioimpedancia consistió en: no tomar líquidos 4 horas antes, no consumir alcohol 24 horas antes de la prueba, o no realizar ningún ejercicio físico, ni tomar cafeína o alimento 4 horas antes de la prueba, entre otras.

\section{Análisis estadístico:}

Para analizar los datos se utilizó el programa estadístico SPSS versión 17.0. Se estudió la normalidad de los mismos mediante la prueba de Kolmogorov-Smirnov. Dado que cumplieron los criterios de normalidad, se utilizó una ANOVA de 2 vías, para así comparar las dos mediciones realizadas por los dos grupos de edad de las mujeres.

Para analizar las diferencias existentes entre ambos grupos de edad, jóvenes y adultas, se utilizó la prueba ANOVA de 1 vía, siendo el factor la edad, y la variable dependiente el porcentaje de cambio (Dif) de las diferentes variables dependientes estudiadas.

Se consideraron como diferencias significativas aquellas con una probabilidad de ser debidas al azar menor al 5\% $(\mathrm{p}<0.05)$.

Los resultados se expresan mediante su media \pm desviación estándar. 


\section{RESULTADOS:}

Los resultados obtenidos se muestran registrados en función de la edad y del programa de actividad física. Además se han insertado columnas llamadas: Diferencia (Dif) expresada en porcentajes (\%). Éstas reflejan los incrementos (+), o descensos (-) producidos en cada una de las variables evaluadas.

Tabla 3

Variables cardiovasculares al inicio y al final del programa de actividad física

\begin{tabular}{|c|c|c|c|c|c|c|}
\hline \multirow{2}{*}{$\begin{array}{l}\text { Mujeres } \\
\text { premenopáusicas }\end{array}$} & \multicolumn{3}{|c|}{ Menores de 35 años: } & \multicolumn{3}{|c|}{ Mayores de 35 años: } \\
\hline & Inicio & Final & Dif (\%) & Inicio & Final & Dif (\%) \\
\hline $\begin{array}{l}\text { Tensión Arterial Sistólica } \\
\text { (mmHg.) }\end{array}$ & $122,88 \pm 12,07$ & $123,50 \pm 13,56$ & $+0,50 \%$ & $137,50 \pm 19,90$ & $130,00 \pm 10,42$ & $-5,45 \%$ \\
\hline $\begin{array}{l}\text { Tensión Arterial Diastólica } \\
\text { (mmHg) }\end{array}$ & $75,00 \pm 14,64$ & $79,00 \pm 7,07$ & $+5,33 \%$ & $95,00 \pm 32,13$ & $84,75 \pm 7,04$ & $\begin{array}{c}-11,05 \% \\
\dagger\end{array}$ \\
\hline Frecuencia Cardíaca (ppm) & $90,75 \pm 22,39$ & $84,00 \pm 11,41$ & $-7,43 \%$ & $82,75 \pm 10,84$ & $76,50 \pm 11,70$ & $-7,55 \%$ \\
\hline
\end{tabular}

† p 0.05 en comparación grupo de "jóvenes" vs grupo de "adultas".

La tabla 3 se muestra que las mujeres menores de 35 años alcanzaron tensiones arteriales mayores al final del programa de actividad física, mientras que la frecuencia cardiaca descendió, pero en ninguno de los valores se alcanzó significación estadística (p>0.05).

Por otro lado, las mujeres mayores de 35 años descendieron todos los parámetros cardiovasculares, pero sin llegar a ser significativos.

Con respecto a las diferencias entre los porcentajes de cambios entre ambos grupos de edad, la tensión arterial diastólica resulta ser la única variable estadísticamente significativa $(p<0.05)$, por su gran descenso en las mujeres mayores de 35 años. 
Tabla 4

Variables de composición corporal al inicio y al final del programa de actividad física

\begin{tabular}{|c|c|c|c|c|c|c|}
\hline \multirow{2}{*}{$\begin{array}{l}\text { Mujeres } \\
\text { premenopáusicas }\end{array}$} & \multicolumn{3}{|c|}{ Menores de 35 años: } & \multicolumn{3}{|c|}{ Mayores de 35 años: } \\
\hline & Inicio & Final & Dif (\%) & Inicio & Final & Dif (\%) \\
\hline Peso (kg) & $60,36 \pm 10,81$ & $56,77 \pm 5,38$ & $-5,94 \%$ & $63,02 \pm 3,96$ & $61,13 \pm 2,73$ & $-2,99 \%$ \\
\hline Masa Libre Grasa (kg) & $45,54 \pm 5,14$ & $43,05 \pm 3,02$ & $-5,46 \%$ & $42,95 \pm 1,08$ & $42,90 \pm 1,40$ & $-0,11 \%$ \\
\hline Masa Libre Grasa (\%) & $76,44 \pm 8,01$ & $76,06 \pm 4,39$ & $-0,49 \%$ & $68,30 \pm 3,83$ & $70,23 \pm 3,23$ & $+2,82 \%$ \\
\hline Masa Grasa (kg) & $14,82 \pm 7,70$ & $13,72 \pm 3,57$ & $-7,42 \%$ & $20,07 \pm 3,71$ & $18,23 \pm 2,68 *$ & $-9,16 \%$ \\
\hline Masa Grasa (\%) & $23,55 \pm 8,01$ & $23,93 \pm 4,39$ & $+1,61 \%$ & $31,70 \pm 3,83$ & $29,76 \pm 3,23 *$ & $-6,11 \%$ \\
\hline Agua Corporal (kg) & $33,33 \pm 3,75$ & $31,53 \pm 2,21$ & $-5,4 \%$ & $31,45 \pm 0,80$ & $31,43 \pm 1,02$ & $-0,06 \%$ \\
\hline Agua Corporal (\%) & $55,93 \pm 5,86$ & $55,75 \pm 3,25$ & $-0,32 \%$ & $50,02 \pm 2,84$ & $51,43 \pm 2,36$ & $+2,81 \%$ \\
\hline Índice de Masa Corporal (kg/m²) & $22,81 \pm 2,49$ & $22,07 \pm 1,26$ & $-3,24 \%$ & $24,87 \pm 2,83$ & $24,06 \pm 2,35$ & $-3,25 \%$ \\
\hline
\end{tabular}

La tabla 4 muestra los parámetros de composición corporal en cada grupo de edad.

En ella se refleja cómo las mujeres menores de 35 años disminuyeron todos los parámetros corporales, excepto el porcentaje de masa grasa, que sufrió un ligero aumento. En ninguno de los casos fue estadísticamente significativo ( $p>0.05)$.

Con lo que respecta a las mujeres mayores de 35 años, excepto la masa libre de grasa (\%) y el agua corporal (\%), el resto de variables disminuyeron, siendo el descenso de la masa grasa (en kg y en \%) estadísticamente significativo ( $\mathrm{p}<0.05)$.

Las diferencias de los porcentajes de cambios entre grupos no fueron estadísticamente significativas.

\section{Discusión:}

En relación con la tensión arterial, y en concreto, al descenso significativo de la tensión diastólica en el grupo de mujeres mayores de 35 años, coincide con los resultados de una investigación donde un programa de actividad física moderado se asoció con la disminución de la tensión arterial diastólica (Mensink, Heerstrass, Neppelenbroek, Schuit, y Bellach, 1997).

Por otro lado, varios estudios también refuerzan estos cambios, pero con la peculiaridad de encontrar mejoras en ambas tensiones (diastólica y sistólica). Estos estudios informan de los efectos positivos en personas que realizan actividad física como Tai-chi (Ko, Tsang, y Chan, 2006) o aquaeróbic (Saavedra, De La Cruz, Escalante, y Rodriguez, 2007); personas activas (Fuster, Rebato, Rosique, y Fernández Lopez, 2008; Palatini y col., 2009); en poblaciones con mayor nivel de actividad física (Gregory, Martorell, Narayan, Ramirez-Zea, y Stein, 2009); con dos programas distintos como la práctica del fútbol o la carrera continua mejora la salud cardiovascular (Krustrup y col., 2010). Estas mejoras se han observado incluso en pacientes con insuficiencia cardíaca crónica tras un programa de entrenamiento aeróbico (Kavanagh y col., 1996). Este descenso podría deberse a que las personas físicamente activas utilizan más los ácidos grasos como fuente energética, disminuyen el nivel de colesterol, mejoran la circulación y disminuyen el nivel de estrés, entre otras, llegando de esta manera a reducir la presión sanguínea. 
También existen algunos estudios que establecen que esta reducción de la tensión arterial diastólica podría ser debida a una mejora en el retorno venoso (Goberna, 2002). Éste señala cómo las mujeres de mediana edad que realizan ejercicio físico mejoran la circulación venosa de retorno y el bombeo muscular, por lo que previene problemas de hipertensión.

En un estudio sobre la adiposidad central, la condición aeróbica y la presión arterial, se informa que en mujeres premenopáusicas hispanas, la presión arterial sistólica fue atribuida en un $25 \%$ a la edad y un $8 \%$ al perímetro de la cintura; mientras que la presión arterial diastólica fue explicada en un $26 \%$ sólo al perímetro de la cintura (Afghani y col., 2004). Estos datos indicaron que la adiposidad central es un predictor importante en la presión arterial en reposos en mujeres hispanas. Esta información vuelve a coincidir con los resultados de la muestra experimental, en la que aparecen las mayores tensiones arteriales en el grupo de mujeres de mayor edad y con mayor porcentaje de masa grasa.

En relación con la composición corporal, y respecto a los cambios significativos en la pérdida de masa grasa en mujeres mayores de 35 años premenopáusicas, coincide con la bibliografía encontrada. Programas similares al de este trabajo alcanzaron los mismos resultados: doce semanas de entrenamiento con clases de aeróbic con step tanto continuo como interválico (Mosher y col., 2005; Isler, 2006).

Según otro estudio realizado con mujeres premenopáusicas obesas, un entrenamiento de tres días a la semana durante seis meses de cuarenta y cinco minutos cada sesión, también reduce los niveles de lípidos (tejido adiposo) e induce una pérdida de peso (Dansou, Kotin, Laleye, Lawani, y Darboux, 2004).

Los motivos podrían atribuirse a las mejoras de los perfiles lipídicos y lipoproteicos que provoca un programa de aeróbic con step (Kin Isler y col., 2001), aunque también se han registrado pérdidas de masa corporal y grasa abdominal con programas de ejercicio al 55\% del consumo máximo de oxígeno (Despres y col., 1991). De hecho, la ganancia de peso es debido al incremento de la masa grasa en personas sedentarias (Schmitz y col., 2007). Estos autores aseguran que las mujeres estadounidenses premenopáusicas (25-44 años) ganan cada año 0.5$1 \mathrm{~kg}$, la mayoría a causa del incremento de la masa grasa. El estudio concluyó que el entrenamiento de fuerza es eficaz para evitar el aumento de porcentaje de grasa corporal en mujeres premenopáusicas.

Sin embargo, otras investigaciones asocian los descensos de masa grasa y el peso corporal no sólo con la actividad física, sino también, con una restricción calórica (Tucker y Bates, 2009; Ito y col., 2001). En relación con la alimentación, Tucker y Thomas (2009) comprobaron que el incremento de ingesta de fibra reduce el riesgo de ganar peso y grasa en mujeres.

Finalmente, cabe destacar la importancia de practicar actividad física en mujeres adultas, ya que les va a permitir mejorar la presión sanguínea y los niveles de grasa. Las mejoras alcanzadas por mujeres adultas son muy favorables, ya que suponen una medida para evitar la hipertensión arterial y otros factores de riesgo de enfermedades cardiovasculares. Con respecto a las mujeres jóvenes, quizás sería necesario un programa de mayor intensidad. Fritz, (2009) dice que la clave para mejorar los niveles corporales es la frecuencia de ejercicio semanal, aunque sin embargo, en otra investigación realizada con niños (Harris, Kuramoto, Schulzer, y Retallack, 2009) se incrementó la cantidad de actividad física en el colegio para prevenir la obesidad infantil, y no mostraron mejoras en el índice de masa corporal. Tampoco encontraron cambios consistentes en otros parámetros de la composición corporal. Sería aconsejable para futuras investigaciones, comparar los efectos de programas con distinta frecuencia semanal en los diferentes parámetros corporales y de tensión arterial, y de esta manera, conocer el grado de importancia de dicha variable, y así tenerla en cuenta en los entrenamientos. 


\section{CONCLUSION:}

Finalmente, este estudio muestra que un programa de ejercicio físico basado en el aeróbic, clases de step y tonificación con mancuernas, a lo largo de cinco meses, realizado durante 3 días alternos a la semana, y con una duración de 70 minutos cada sesión, mejora factores de riesgo cardiovasculares como la hipertensión arterial y la composición corporal, por su reducción en mayor medida en la tensión arterial diastólica y en la masa grasa en el grupo de mujeres mayores de 35 años premenopáusicas.

\section{REFERENCIAS:}

Afghani, A., Abbott, A. V., Wiswell, R. A., Jaque, S. V., Gleckner, C., Schroeder, E. T., y col., (2004). Central adiposity, aerobic fitness, and blood pressure in premenopausal Hispanic women. International Journal of Sports Medicine, 25(8), 599-606.

Anthony, D., Baggott, R., Tanner, J., Jones, K., Evans, H., Perkins, G., y cols. (2012). Health, lifestyle, belief and knowledge differences between two ethnic groups with specific reference to tobacco, diet and physical activity. Journal of Advanced Nursing.

Arsenault, B. J., Rana, J. S., Lemieux, I., Despres, J. P., Kastelein, J. J., Boekholdt, S. M., y cols. (2009). Physical inactivity, abdominal obesity and risk of coronary heart disease in apparently healthy men and women. International Journal of Obesity (London), 34(2), 340-347.

Cowley, A. W., Jr., Dzau, V., Buttrick, P., Cooke, J., Devereux, R. B., Grines, C. L., y col., (1992). Working group on noncoronary cardiovascular disease and exercise in women. Medicine \& Science in Sports \& Exercise, 24(6 Suppl), S277-287.

Dansou, P., Kotin, M. L., Laleye, A., Lawani, M., y Darboux, R. (2004). Effects of physical activity on adipose tissue cellularity in premenopausal obese women in Benin. Sante, 14(3), 183-186.

Díaz Sánchez M. E. (1999). Trabajo de Revisión: Biotipo y climaterio femenino. Revista Cubana de Medicina General Integral. v. 15 n.3

Despres, J. P., Pouliot, M. C., Moorjani, S., Nadeau, A., Tremblay, A., Lupien, P. J., y col., (1991). Loss of abdominal fat and metabolic response to exercise training in obese women. American Journal of Physiology, 261(2 Pt 1), E159-167.

Fritz, P. (2009). [Health development program package and its effectiveness on the students in high schools and universities.]. Orv Hetil, 150(27), 1281-1288.

Fuster, V., Rebato, E., Rosique, J., y Fernandez Lopez, J. R. (2008). Physical activity related to forced vital capacity and strength performance in a sample of young males and females. Collegium Antropologicum, 32(1), 53-60.

Goberna, J. (2002). Promoción de la salud en el climaterio. Matronas Profesión, 8, 412. 
Godfrey, J. R., y Nelson, M. E. (2009). Toward optimal health: promoting physical activity in women. Journal of Women's Health (Larchmt), 18(3), 295-298.

Gregory, C. O., Martorell, R., Narayan, K. M., Ramirez-Zea, M., y Stein, A. D. (2009). Five-year changes in adiposity and cardio-metabolic risk factors among Guatemalan young adults. Public Health Nutrition, 12(2), 228-235.

Harris, K. C., Kuramoto, L. K., Schulzer, M., y Retallack, J. E. (2009). Effect of school-based physical activity interventions on body mass index in children: a meta-analysis. Canadian Medical Association Journal, 180(7), 719-726.

Hauner, H. (1995). Nutrition and metabolic syndrome. Internist (Berl), 36(11), 10401045.

Henry-Okafor, Q., Cowan, P. A., Wicks, M. N., Rice, M., Husch, D. S., y Khoo, M. S. (2011). Effect of obesity on cardiovascular disease risk factors in african american women. Biological Research For Nursing, 14(2), 171-179.

Hjern, F., Wolk, A., y Hakansson, N. (2011). Obesity, physical inactivity, and colonic diverticular disease requiring hospitalization in women: a prospective cohort study. The American Journal of Gastroenterology, 107(2), 296-302.

Isler, A. K. (2006). Lipid and lipoprotein changes in premenstrual women following step aerobic dance training. International Journal of Sports Medicine, 27(4), 343.

Ito, H., Ohshima, A., Tsuzuki, M., Ohto, N., Yanagawa, M., Maruyama, T., y col., (2001). Effects of increased physical activity and mild calorie restriction on heart rate variability in obese women. Japanese Heart Journal, 42(4), 459-469.

Kalman, D., Colker, C. M., Wilets, I., Roufs, J. B., y Antonio, J. (1999). The effects of pyruvate supplementation on body composition in overweight individuals. Nutrition, 15(5), 337-340.

Kang, H. S., Matsuo, T., y Suzuki, M. (2004). Effects of light resistance exercise using dumbbells and rubber band with mild energy restriction on body composition and physical fitness in obese Korean women. Asia Pacific Journal of Clinical Nutrition, 13(3), 242-247.

Kavanagh, T., Myers, M. G., Baigrie, R. S., Mertens, D. J., Sawyer, P., y Shephard, R. J. (1996). Quality of life and cardiorespiratory function in chronic heart failure: effects of 12 months' aerobic training. Heart, 76(1), 42-49.

Kaye, S. A., Folsom, A. R., Prineas, R. J., Potter, J. D., y Gapstur, S. M. (1990). The association of body fat distribution with lifestyle and reproductive factors in a population study of postmenopausal women. International Journal of Obesity, 14(7), 583-591. 
Kin Isler, A., Kosar, S. N., y Korkusuz, F. (2001). Effects of step aerobics and aerobic dancing on serum lipids and lipoproteins. Journal of Sports Medicine and Physical Fitness, 41(3), 380-385.

Kizer, J. R., Biggs, M. L., Ix, J. H., Mukamal, K. J., Zieman, S. J., de Boer, I. H., y cols. (2010). Measures of adiposity and future risk of ischemic stroke and coronary heart disease in older men and women. American Journal of Epidemiology, 173(1), 10-25.

Ko, G. T., Tsang, P. C., y Chan, H. C. (2006). A 10-week Tai-Chi program improved the blood pressure, lipid profile and SF-36 scores in Hong Kong Chinese women. Medical Science Monitor, 12(5), CR196-199.

Krustrup, P., Hansen, P. R., Randers, M. B., Nybo, L., Martone, D., Andersen, L. J., y col. (2010). Beneficial effects of recreational football on the cardiovascular risk profile in untrained premenopausal women. Scandinavian Journal of Medicicine \& Science in Sports, 20 Suppl 1, 40-49.

Leyk, D., Ruther, T., Wunderlich, M., Heiss, A., Kuchmeister, G., Piekarski, C., y col., (2008). Sporting Activity, Prevalence of Overweight, and Risk Factors: Cross-Sectional Study of More Than 12500 Participants Aged 16 to 25 Years. Deutsches Arzteblatt International, 105(46), 793-800.

Mensink, G. B., Heerstrass, D. W., Neppelenbroek, S. E., Schuit, A. J., y Bellach, B. M. (1997). Intensity, duration, and frequency of physical activity and coronary risk factors. Medicine and Science in Sports and Exercise, 29(9), 1192-1198.

Montalcini, T., Gorgone, G., Garzaniti, A., Gazzaruso, C., y Pujia, A. (2010). Artery remodeling and abdominal adiposity in nonobese postmenopausal women. European Journal of Clinical Nutrition, 64(9), 1022-1024.

Mosher, P. E., Ferguson, M. A., y Arnold, R. O. (2005). Lipid and lipoprotein changes in premenstrual women following step aerobic dance training. International Journal of Sports Medicine, 26(8), 669-674.

Oguma, Y., Sesso, H. D., Paffenbarger, R. S., Jr., y Lee, I. M. (2002). Physical activity and all cause mortality in women: a review of the evidence. British Journal of Sports Medicine, 36(3), 162-172.

Owens, J. F., Matthews, K. A., Wing, R. R., y Kuller, L. H. (1990). Physical activity and cardiovascular risk: a cross-sectional study of middle-aged premenopausal women. Preventive Medicine, 19(2), 147-157.

Palatini, P., Visentin, P., Dorigatti, F., Guarnieri, C., Santonastaso, M., Cozzio, S., y col., (2009). Regular physical activity prevents development of left ventricular hypertrophy in hypertension. European Heart Journal, 30(2), 225-232.

Rana, J. S., Li, T. Y., Manson, J. E., y Hu, F. B. (2007). Adiposity compared with physical inactivity and risk of type 2 diabetes in women. Diabetes Care, 30(1), 53-58. 
URL www.una.ac.cr/mhsalud

Saavedra, J. M., De La Cruz, E., Escalante, Y., y Rodriguez, F. A. (2007). Influence of a medium-impact aquaerobic program on health-related quality of life and fitness level in healthy adult females. Journal of Sports Medicine and Physical Fitness, 47(4), 468-474.

Schmitz, K. H., Hannan, P. J., Stovitz, S. D., Bryan, C. J., Warren, M., y Jensen, M. D. (2007). Strength training and adiposity in premenopausal women: strong, healthy, and empowered study. American Journal of Clinical Nutrition, 86(3), 566-572.

Tucker, L. A., y Bates, L. (2009). Restrained eating and risk of gaining weight and body fat in middle-aged women: a 3-year prospective study. American Journal of Health Promotion, 23(3), 187-194.

Tucker, L. A., y Thomas, K. S. (2009). Increasing total fiber intake reduces risk of weight and fat gains in women. Journal of Nutrition, 139(3), 576-581.

Van Roie, E., Delecluse, C., Opdenacker, J., De Bock, K., Kennis, E., y Boen, F. (2010). Effectiveness of a lifestyle physical activity versus a structured exercise intervention in older adults. Journal of Aging and Physical Activity, 18(3), 335-352.

Wang, C. Y., Yeh, C. J., Wang, C. W., Wang, C. F., y Lin, Y. L. (2010). The health benefits following regular ongoing exercise lifestyle in independent community-dwelling older Taiwanese adults. Australasian Journal on Ageing, 30(1), 22-26.

Fecha de recepción: 28 de noviembre de 2011.

Fecha de aceptación: 17 de mayo de 2012.

Fecha de publicación: 31 de julio del 2012. 